\title{
REAÇÕES ADVERSAS E ANAFILÁTICAS APÓS O USO DE PENICILINA BENZATINA EM GESTANTES COM SÍFILIS: REVISÃO INTEGRATIVA
}

\author{
ADVERSE AND ANAPHYLACTIC REACTIONS AFTER THE USE OF \\ BENZATHINE PENICILLIN IN PREGNANT WOMEN WITH SYPHILIS: \\ INTEGRATIVE REVIEW
}

Jaiza Sousa Penha1, Poliana Pereira Costa Rabelo², Geysa Santos Góis Lopes $^{3^{*}}$, Sara Maria Ferreira de Sousa ${ }^{4}$, Raires Castro de Sá ${ }^{5}$, Cindy Rebouças Palmeira6$^{6}$, Isaura Letícia Tavares Palmeira Rolim ${ }^{7}$

\begin{abstract}
${ }^{1}$ Mestranda em Enfermagem pela Universidade Federal do Maranhão.
2Doutorado em Enfermagem em Saúde Pública pela Universidade de São Paulo. Docente da Universidade Federal do Maranhão.

${ }^{3}$ Mestre em Enfermagem pela Universidade Federal do Maranhão

${ }^{4}$ Mestranda em Saúde e Ambiente pela Universidade Federal do Maranhão.

${ }^{5}$ Especialista em Unidade de Terapia Intensiva pela Faculdade Redentor.

${ }^{6}$ Mestranda em Ciências da Cidade pela Universidade de Fortaleza.

${ }^{7}$ Doutorado em Enfermagem pela Universidade Federal do Ceará. Docente da Universidade Federal do Maranhão.
\end{abstract}

*Av. dos Portugueses, no 1966 - Vila Bacanga, São Luís/MA. CEP 65080-805. E-mail:
geysagois@hotmail.com

Submetido em: 13/01/2020; Aceito em: 09/06/2020.

\section{RESUMO}

A sífilis na gestação consiste em um relevante problema de saúde pública, podendo causar graves problemas na saúde da criança. No tratamento, o uso da penicilina benzatina possui a sua segurança questionada devido à possibilidade de ocorrência de reações adversas. Trata-se de uma revisão integrativa da literatura que buscou analisar a produção científica acerca da ocorrência de reações adversas e anafiláticas após o uso de penicilina benzatina no tratamento de sífilis em gestantes a partir de publicações indexadas nas bases de dados Medical Literature Analysis and Retrieval System Online (MEDLINE), Scientific Eletronic Library Online (SCIELO), Literatura Latino Americana e do Caribe em Ciências da Saúde (LILACS) e Base de dados em Enfermagem (BDENF), entre dezembro de 2018 a dezembro de 2019. Foram incluídos na amostra seis estudos. Reafirmou-se a administração com segurança da penicilina benzatina na rede básica de saúde e confirmou-se que pacientes sifilíticas deixam de receber tratamento adequado pelo medo dos profissionais. O uso da penicilina é eleito como o mais resolutivo a fim de evitar as consequências da sífilis gestacional para mãe e concepto. Reitera-se a importância do aparato tecnológico necessário em casos emergenciais, profissionais capacitados e a articulação das redes de saúde.

Palavras-chave: Anafilaxia. Gestante. Penicilinas. Reações Adversas a Medicamentos. 


\section{ABSTRACT}

The syphilis in pregnancy is a major public health problem, which can cause serious problems in child health. In the treatment, the use of benzathine penicillin has its safety questioned due to the possibility of occurrence of adverse reactions. This is an integrative literature review that sought to analyze the scientific production about the occurrence of adverse and anaphylactic reactions after the use of benzathine penicillin in the treatment of syphilis in pregnant women from publications indexed in databases Medical Literature Analysis and Retrieval System Online (MEDLINE), Scientific Electronic Library Online (SCIELO), Latin American and Caribbean Health Sciences Literature (LILACS) and the Nursing Database (BDENF), from December 2018 to December 2019. The sample included six studies. The safe administration of benzathine penicillin in the basic health network is reaffirmed and the syphilitic patients fail to receive appropriate treatment due to professionals' fear. The use of penicillin is elected as the most decisive in order to avoid the consequences of gestational syphilis for mother and fetus. The importance of the technological apparatus required in emergency cases is reiterated, in addition to qualified professionals and the articulation of health networks.

Keywords: Anaphylaxis. Pregnant women. Penicillin. Adverse Reactions to Medications.

\section{INTRODUÇÃO}

A sífilis caracteriza-se como uma doença infecciosa bacteriana, transmitida a partir do contato com seu agente etiológico o Treponema pallidum, podendo afetar diversos órgãos e sistemas, com manifestações que ocorrem em fases distintas e intermitentes. Destaca-se a possibilidade de infecção da doença entre mãe e bebê, intitulada sífilis congênita (SC), com consequências graves ao feto e considerada importante causa de abortos, natimortos, prematuridade, hidrópsia fetal, mortalidade neonatal e morbidades em lactentes (BRACCIO; SHARLAND; LADHANI, 2016).

Consiste em um relevante problema de saúde pública e, segundo a organização mundial da saúde (OMS), 12 milhões de pessoas são infectadas no mundo a cada ano, sendo as regiões mais afetadas a África Subsaariana, a América Latina e o sudeste Asiático, apresentando maior incidência em países emergentes com cerca de 90\% dos casos (COHEN et al., 2013; DE AQUINO; GONÇALVES, 2016).

Diante da variedade de sinais e sintomas que circundam a sífilis, é imprescindível que os profissionais estejam capacitados para identificar a doença nas suas diferentes fases clínicas, realizando o diagnóstico da doença em tempo hábil, contribuindo, então, para o tratamento adequado (AVELLEIRA; BONTTINO, 2006; KALININ, PASSARELLI NETO; PASSARELLI, 2016)

Frente a um resultado positivo, o tratamento deve ser iniciado de acordo com o estágio clínico e a droga de escolha atualmente é a penicilina benzatina. Estudos mostram que a sua eficácia é de $99,7 \%$ para tratamento materno e $98,2 \%$ para prevenção da transmissão vertical da doença em todos os estágios 
da sífilis. As poucas evidências que relatam falha na terapia estão associadas a pacientes HIV positivas e, mesmo assim, não evidenciam resistência do Treponema pallidum à penicilina (QUÉTEL; BRADDOCK; PIKE, 1990; DE SANTIS et al., 2012).

Apesar disso, as penicilinas são medicamentos que constantemente tem sua segurança questionada, principalmente devido à ocorrência de reações adversas. Estima-se que a prevalência desses casos seja em torno de $2 \%$ por curso de tratamento. Entretanto, cerca de $90 \%$ dos indivíduos que relatam hipersensibilidade à penicilina não são alérgicos (FELIX; KUSCHNIR, 2011; FELIX; MALAMAN; ENSINA, 2014).

Por esses motivos o Ministério da Saúde e alguns autores afirmam que há a resistência por parte dos profissionais de saúde na administração da penicilina em gestantes, especialmente na rede básica, por medo de reações adversas e/ou anafiláticas. Associado a isso, afirmam ainda que há falta de infraestrutura adequada para atenderem a essas demandas específicas (BRASIL, 2018).

Tomando como justificativa a epidemia de sífilis e o seu difícil controle por causas diversas que variam desde o sexo desprotegido até as falhas no tratamento, faz-se importante a realização de estudos que buscam embasar a prática técnico-científica, comprovados por pesquisas anteriores e baseadas em evidências, contribuindo para a quebra da cadeia de transmissão, tendo em vista o grande impacto socioeconômico da doença.

Desse modo, este estudo objetivou analisar a produção científica acerca da ocorrência de reações adversas e anafiláticas após o uso de penicilina benzatina no tratamento de sífilis em gestantes.

\section{METODOLOGIA}

Trata-se de uma revisão integrativa que teve como questão norteadora: qual a ocorrência de reações adversas e anafiláticas após o uso de Penicilina Benzatina em pacientes gestantes em tratamento de sífilis?

A estratégia de identificação e seleção dos estudos foi a busca de publicações indexadas nas bases de dados eletrônicas: Medical Literature Analysis and Retrieval System Online (MEDLINE), Scientific Eletronic Library Online (SCIELO), Literatura Latino Americana e do Caribe em Ciências da Saúde (LILACS) e Base de dados em Enfermagem (BDENF), através dos Descritores em Saúde (DecS): Penicilinas. Reações Adversas e Efeitos Colaterais Relacionados a Medicamentos. Anafilaxia. Gestantes. A coleta ocorreu no período de dezembro de 2018 a dezembro de 2019 utilizando-se os Descritores em Ciências da Saúde (DeCS): "Penicilinas", "Efeitos Colaterais e Reações Adversas Relacionados a Medicamentos", "Anafilaxia" e "Gestantes", isolados e associados, nos idiomas português ou inglês, para verificação do título, resumo ou assunto de acordo com a base de dados. Foram incluídos na amostra os estudos que contemplaram a pergunta norteadora, publicados no período de 2008 a 2019, nos idiomas português, inglês e espanhol, disponíveis online na íntegra.

Foram excluídos aqueles que não atenderam aos objetivos estabelecidos ou que estiveram no formato de editoriais, cartas ao editor ou opiniões de 
especialistas, bem como os trabalhos que se encontraram duplicados e que se distanciaram da temática a partir da leitura dos resumos.

Posteriormente, os artigos foram organizados e categorizados por meio de um quadro sinóptico. Ao realizar a busca na base de dados, os artigos científicos identificados foram avaliados inicialmente por meio da análise dos títulos e resumos, considerando a pergunta norteadora e obedecendo aos critérios de inclusão.

Após esse processo, procedeu-se à leitura na íntegra dos artigos selecionados na etapa anterior e daqueles cujos títulos e resumos foram insuficientes para a seleção inicial. A leitura foi realizada de modo criterioso para a extração dos dados.

A autoria dos artigos selecionados foi assegurada, sendo os mesmos referenciados ao longo do texto. Considerando tratar-se de uma revisão integrativa e, portanto, não envolve seres humanos, tornou-se dispensável a aprovação do projeto no Comitê de Ética em Pesquisa.

\section{DESENVOLVIMENTO}

Foram incluídos seis estudos dentre os mais de 2 mil identificados. Foram empregadas etapas sucessivas que permitiram filtrar a amostra, de modo a realizar a redação final desta pesquisa. A Figura 1 apresenta o fluxograma que detalha o percurso, busca, identificação, elegibilidade e seleção.

Figura 1 - Etapas de busca e seleção dos estudos selecionados.

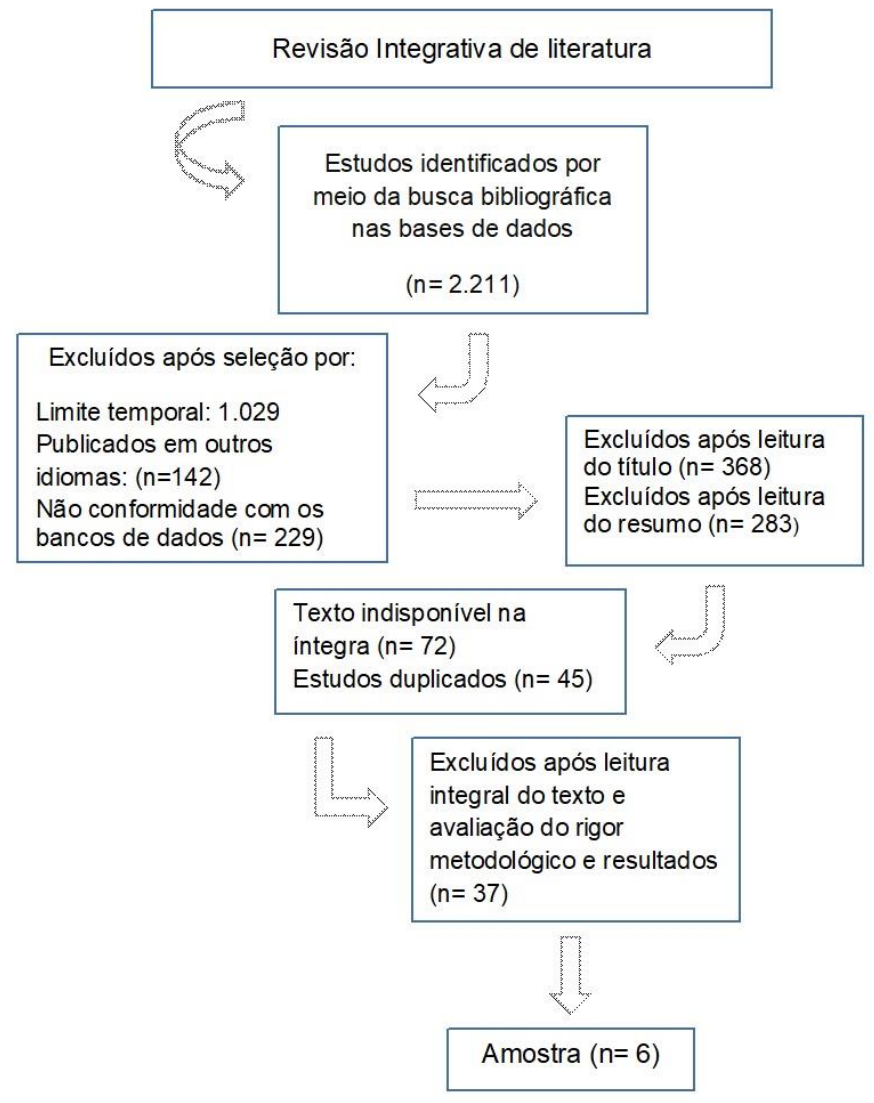

Fonte: os autores. 
As pesquisas foram realizadas nas bases de dados supracitadas, utilizando ampla abordagem nos materiais disponíveis de maneira integral e gratuito, e que utilizaram metodologias consistentes para compilar este estudo. De acordo com a base de dados, foram encontrados artigos na Scielo, Medline e Lilacs, nos idiomas português e inglês.

A maioria dos estudos elegíveis que se dedicaram a investigar as reações adversas, efeitos colaterais e anafilaxia após o uso de penicilina benzatina não tiveram como população-alvo as gestantes com VDRL positivo, e sim as ocorrências após a administração do medicamento em pacientes em geral, o que permite afirmar que a maioria das pesquisas detalha de maneira mais abrangente dados como perfil socioeconômico, diagnóstico e tratamento de mulheres com sífilis, além de abordagens que investigam a adesão do parceiro ao tratamento.

O Quadro 1 descreve de maneira resumida os autores/ano, título do artigo, resultados e conclusão dos estudos que foram selecionados para esta análise.

\section{O uso da penicilina nas unidades básicas de saúde}

Vaz (2008) objetivou em seu estudo apresentar a descrição e propor diálogo sobre os critérios de notificação da sífilis congênita no Brasil. Quando faz menção à terapêutica empregada na doença em gestantes, reafirma ser este o melhor tratamento, sendo a penicilina benzatina a droga de escolha, que deve ser administrada até 30 dias antes do parto. Quando discutida as reações adversas e anafilaxia em pacientes sabidamente alérgicas, a pesquisa menciona uma droga coadjuvante, a eritromicina, com limitação em virtude de tratar somente a mulher e não o feto.

No entanto, um estudo considera inadequado o tratamento à gestante realizado com qualquer outro medicamento que não seja a penicilina, elevando a ocorrência da sífilis congênita (TSAl et al., 2019). O estudo também afirma que todas as unidades avaliadas tinham material o mínimo suficiente e pessoal treinado para atender gestantes que reagem adversamente ao emprego da penicilina benzatina, e recomenda que a assistência, nos casos de anafilaxia, seja imediata (VAZ, 2008).

O estudo de Ramalho (2016) corrobora com o anterior ao apresentar dados substanciais em relação ao emprego da penicilina em gestantes e a possibilidade de reações adversas acontecerem, afirmando que os profissionais referiram limitação na aplicação da droga devido à falta de insumos suficientes para utilização em casos necessários e ainda a inexperiência no manejo da sífilis. Os enfermeiros realizaram a maioria dos diagnósticos da doença, porém, apenas $9,2 \%$ da categoria se propôs a realizar o seguimento e tratamento dos casos, sendo, então, realizados pelo profissional médico em mais de $90 \%$ das situações.

Reafirmando o exposto, foi observado que, num Estado do nordeste brasileiro, a maioria dos pacientes sifilíticos era encaminhada para outras unidades, sendo essas de maior complexidade, ou para o profissional médico, em razão do entendimento de que a prescrição de fármacos é um ato exclusivo dos mesmos (DANTAS, 2008). 
Quadro 1. Identificação de pesquisas quanto às reações adversas e anafiláticas após o uso de penicilina benzatina em gestantes com sífilis, incluindo autoria, ano de publicação, tipo de pesquisa, resultado e conclusão. São Luís, Maranhão, 2019.

\begin{tabular}{|c|c|c|c|}
\hline AUTOR / ANO & TÍTULO DO ARTIGO & RESULTADO & CONCLUSÃO \\
\hline $\begin{array}{l}\text { Rezende; Barbosa } \\
(2015)\end{array}$ & $\begin{array}{l}\text { A sífilis congênita como indicador } \\
\text { da assistência de pré-natal no } \\
\text { estado de Goiás }\end{array}$ & $\begin{array}{l}3382 \text { gestantes foram diagnosticadas } \\
\text { com sífilis entre } 2009 \text { e } 2012 ; 12,6 \% \\
\text { não realizou tratamento no período } \\
\text { avaliado; } 5,7 \% \text { das gestantes } \\
\text { realizaram outro esquema de } \\
\text { tratamento diferente do preconizado. }\end{array}$ & $\begin{array}{l}\text { Não foram registrados casos de } \\
\text { reações, anafilaxia ou efeitos } \\
\text { adversos. }\end{array}$ \\
\hline $\begin{array}{l}\text { Guanabara et al. } \\
(2017)\end{array}$ & $\begin{array}{l}\text { Access of pregnant women to } \\
\text { technologies for the prevention } \\
\text { and control of congenital syphilis } \\
\text { in Fortaleza-Ceará, Brazil. }\end{array}$ & $\begin{array}{l}\text { As farmácias das unidades tinham } \\
\text { estoque de penicilina benzatina em } \\
\text { quantidade suficiente, e eram inclusive } \\
\text { disponibilizadas às gestantes com } \\
\text { VDRL positivo. Porém, as mesmas } \\
\text { eram encaminhadas para serviços de } \\
\text { maior complexidade para } \\
\text { administração da medicação devido às } \\
\text { más condições da unidade atender às } \\
\text { reações adversas e anafiláticas. }\end{array}$ & $\begin{array}{l}\text { Foi unânime a resistência e até } \\
\text { recusa por parte dos profissionais em } \\
\text { atender a recomendação de aplicar a } \\
\text { penicilina na atençâo primária em } \\
\text { saúde. A alegativa está pautada na } \\
\text { possibilidade de reações adversas } \\
\text { provocadas pelo medicamento e na } \\
\text { falta de condições materiais e de } \\
\text { insumos para manejar os casos de } \\
\text { anafilaxia. }\end{array}$ \\
\hline Galvão (2014) & $\begin{array}{c}\text { Segurança da benzilpenicilina } \\
\text { benzatina na atenção básica } \\
\text { em saúde. }\end{array}$ & $\begin{array}{l}\text { Foram incluídos } 13 \text { estudos. Não foram } \\
\text { relatadas reações adversas graves ou } \\
\text { morte nas } 1.244 \text { gestantes avaliadas, } \\
\text { em cinco estudos de coorte. Na } \\
\text { população geral, foram observados } 54 \\
\text { casos de anafilaxia em } 2.028 .982 \\
\text { pacientes tratados. }\end{array}$ & $\begin{array}{l}\text { A incidência de reações adversas } \\
\text { graves relatadas nos estudos } \\
\text { utilizados para a elaboração desta } \\
\text { meta-análise foi muito baixa. A } \\
\text { terapia à base de penicilina é conduta } \\
\text { de escolha para importantes doenças } \\
\text { prevalentes na atenção básica em } \\
\text { saúde, o que sugere que os possíveis } \\
\text { riscos não ultrapassam seus } \\
\text { benefícios. }\end{array}$ \\
\hline
\end{tabular}


Quadro 1, cont.

\begin{tabular}{|c|c|c|c|}
\hline $\begin{array}{l}\text { Grossi et al. } \\
(2011)\end{array}$ & $\begin{array}{c}\text { Reação de Jarisch-Herxheimer } \\
\text { em gestante com sífilis: relato } \\
\text { de caso. }\end{array}$ & $\begin{array}{l}\text { Foi prescrito penicilina G benzatina } \\
2.400 .000 \text { UI (IM), para tratamento da } \\
\text { sífilis. Quatro horas após a } \\
\text { administração da penicilina, a paciente } \\
\text { apresentou mialgia intensa, artralgia e } \\
\text { febre aferida de } 39^{\circ} \mathrm{C} \text { e mal-estar geral, } \\
\text { sugerindo Reação de Jarisch- } \\
\text { Herxheimer. }\end{array}$ & $\begin{array}{l}\text { É aconselhado que as gestantes } \\
\text { estejam cientes dos sinais e } \\
\text { sintomas dessa reação e que } \\
\text { relatem imediatamente ao obstetra } \\
\text { o aparecimento dos mesmos a fim } \\
\text { de minimizar a morbidade e a } \\
\text { mortalidade associadas a essa } \\
\text { condição. A RJH pode ser } \\
\text { confundida com hipersensibilidade } \\
\text { à penicilina. }\end{array}$ \\
\hline Vaz (2008) & $\begin{array}{c}\text { Sífilis congênita: critérios de } \\
\text { notificação. }\end{array}$ & $\begin{array}{l}\text { Nos casos de reação alérgica ou } \\
\text { anafilaxia comprovada pode-se tratar } \\
\text { com estearato de eritromicina não } \\
\text { sendo tratamento considerado } \\
\text { adequado, pois não trata o feto. Após o } \\
\text { tratamento a gestante deve realizar } \\
\text { controle mensal com teste de VDRL e } \\
\text { novo tratamento instituído no caso de } \\
\text { reinfecção ou aumento dos títulos no } \\
\text { VDRL. }\end{array}$ & $\begin{array}{l}\text { Os profissionais que atuam no pré- } \\
\text { natal devem ter conhecimento de tais } \\
\text { recomendações, para atingir } \\
\text { resultados de qualidade de } \\
\text { assistência que permitam erradicar a } \\
\text { sífilis, e principalmente a congênita } \\
\text { em todos os municípios brasileiros. }\end{array}$ \\
\hline Ramalho (2016) & $\begin{array}{l}\text { Avaliação da assistência pré- } \\
\text { natal com ênfase na sífilis } \\
\text { gestacional na estratégia de } \\
\text { Saúde da Família do Recife. }\end{array}$ & $\begin{array}{l}\text { Dentre os profissionais que não faziam } \\
\text { o tratamento na unidade, o principal } \\
\text { motivo alegado foi o medo das } \\
\text { possíveis reações anafiláticas } \\
\text { decorrentes da penicilina. }\end{array}$ & $\begin{array}{l}\text { A droga mais prescrita para o } \\
\text { tratamento foi a penicilina benzatina } \\
\text { em esquema para sífilis terciária. } \\
\text { Embora a literatura reporte as } \\
\text { reações anafiláticas como evento } \\
\text { raro, algumas USF não } \\
\text { administravam o fármaco em suas } \\
\text { dependências pelo medo dessas } \\
\text { reações. }\end{array}$ \\
\hline
\end{tabular}

Fonte: os autores. 
Em contrapartida, o Conselho Federal de Enfermagem do Brasil, em 2017, lançou uma nota técnica que ampara a equipe de enfermagem com relação ao tratamento e manejo da sífilis, permitindo a prescrição do tratamento de acordo com protocolos do ministério da saúde, além da aplicação da penicilina nas unidades básicas. A decisão reforça ainda o importante papel da Enfermagem na condução das infecções sexualmente transmissíveis, bem como a participação conjunta das equipes multiprofissionais (COFEN, 2017).

Guanabara et al. (2017) ao avaliar o acesso de gestantes às tecnologias para prevenção e controle da sífilis congênita, encontrou resultados similares, onde a resistência e até recusa por parte dos profissionais em administrar a penicilina benzatina foi outro problema identificado e a alegação era a possibilidade de reação anafilática. Entre os entrevistados neste estudo, um médico e uma enfermeira responderam:

\begin{abstract}
"Eu não autorizo aplicação da penicilina porque às vezes falta medicação no caso de alguma reação alérgica. A gente não tem como fazer medicação venosa, nem intramuscular porque não tem material de suporte" (médico). "Os profissionais não se sentem seguros de fazer a penicilina. Não tem equipamento, não tem bala de oxigênio, não tem medicação de urgência para atender uma possível ocorrência" (enfermeira).
\end{abstract}

As unidades de saúde avaliadas pela pesquisa anteriormente citada tinham estoque de penicilina benzatina suficiente, de tal maneira que a medicação, em suma, era liberada às gestantes com VDRL positivo para iniciarem o tratamento em unidades de maior complexidade. A principal justificativa dos entrevistados se baseou na falta de medicações, equipamentos e estrutura inadequada para 0 atendimento em caso de reações anafiláticas graves (GUANABARA et al., 2017).

Concordando com esse cenário, Nunes et al. (2018) publicaram em estudo recente que a incidência de sífilis gestacional aumentou de 2,8 para 9,5 a cada mil nascidos vivos, e a sífilis congênita, de 0,3 para 2,5/mil nascidos vivos, sendo identificados os maiores índices onde a cobertura da Estratégia Saúde da Família foi baixa. Portanto, as unidades básicas de saúde, que fazem parte desse contexto, precisam estar preparadas com recursos humanos capacitados, insumos e equipamentos necessários para o diagnóstico, o tratamento e 0 seguimento adequado dos casos (NUNES et al., 2018).

Assim, o Ministério da Saúde reafirma que a penicilina benzatina pode ser administrada na rede básica de saúde com segurança e confirma que, em muitos casos, pacientes sifilíticas deixam de receber tratamento adequado pelo medo dos profissionais quando na aplicação do fármaco, o que contribui para a manutenção da cadeia de transmissão da doença (BRASIL, 2018).

\title{
A ocorrência de reações adversas e anafiláticas
}

Ao analisar as ocorrências de reações adversas e/ou anafiláticas, Rezende e Barbosa (2015) apresentaram em uma análise descritiva dados da incidência de sífilis congênita no estado de Goiás de 2009 a 2012. No período avaliado, houve 3.382 casos de sífilis em gestantes diagnosticados. Dentre elas, um percentual de $12,6 \%$ não realizou tratamento no período avaliado; $5,7 \%$ das 
gestantes realizaram outro esquema de tratamento, diferente do preconizado; e não foram descritos casos de ocorrência de reação adversa ou anafiláticas.

Já a pesquisa realizada por Galvão et al. (2014) que avaliaram a segurança da benzilpenicilina benzatina na atenção básica em saúde, através de uma revisão sistemática, concluiu que, entre as 1.244 gestantes que utilizaram a droga, não foram relatados casos de reações adversas. Entre a população geral, dos 2.028.982 pacientes tratados houve 54 ocorrências de anafilaxia. Portanto, de acordo com essas estimativas, a chance de reação alérgica grave pode ocorrer entre 0 a 3 casos a cada 100.000 pessoas que entrem em contato com a medicação.

Esses dados reforçam as indicações de outros autores e do Ministério da Saúde que, através da Comissão Nacional de Incorporação de Tecnologias no SUS (CONITEC), indica que a penicilina é altamente eficaz no tratamento da sífilis em gestantes e na prevenção da forma congênita da doença, tendo garantida a segurança do seu uso em razão do baixo risco para reações adversas e anafiláticas, podendo ser manejada na rede básica de saúde no país (FELIX; KUSCHNIR, 2011; BRASIL, 2018).

Nesse contexto, dentre as possibilidades de reações à medicação, a mais comum é denominada de Jarisch-Herxheimer, investigada em um estudo de caso incluído nesta pesquisa. É caracterizada por picos febris agudos, que podem ocorrer em limite de tempo entre 12 a 24 horas após o emprego do tratamento, possui alta incidência (cerca de $80 \%$ ), sendo geralmente acompanhada de cefaleia, mialgia, rigidez, sudorese, hipotensão e exantema progressivo que podem ser confundidos como uma reação de hipersensibilidade ao fármaco e, portanto, necessitam de uma boa condução dos casos (GROSSI et al., 2011; TSIMIS; SHEFFIELD, 2017; HENZ, 2018).

Quando acontecem, as reações adversas à penicilina costumam atingir mais as mulheres, entre 20 e 49 anos de idade e pacientes com oposição prévia à substância, quando empregada novamente. A via de administração e a frequência de uso da droga também são variáveis importantes, destacando-se a via parenteral e as exposições intermitentes e repetidas (FELIX; MALAMAN; ENSINA, 2014).

Nos casos em que há chances ou a confirmação de alergia a penicilina, a recomendação é a dessensibilização da droga, em razão do custo/efetividade do tratamento quando realizado de forma adequada. O procedimento é feito de acordo com protocolos específicos e o objetivo é induzir o indivíduo a um estado de tolerância que é transitório, por meio de doses pequenas administradas em intervalos regulares, até que a dose alvo seja atingida. Estudo recente, onde a dessensibilização foi feita em dez gestantes sifilíticas, demonstrou que todas obtiveram boa resposta, sem reações adversas, confirmando a segurança do método (CAIADO, 2016; DALLÉ et al., 2018).

Quando não for possível realizar a dessensibilização em pacientes alérgicas, não existe uma segunda opção. Apesar de ser utilizada como alternativa em pacientes adultos, a ceftriaxona não pode ser utilizada em grávidas, em razão da toxicidade ao feto e por não atravessar a barreira transplacentária. Desse modo, o tratamento seria considerado inadequado, devendo a usuária ser monitorada, uma vez que aumentam os riscos da forma congênita da doença (SHENOY et al., 2019; TORRES et al., 2019). 
Portanto, a penicilina benzatina é considerada a melhor opção e com capacidade resolutiva de se evitarem as consequências que a sífilis gestacional pode causar na saúde da mãe e do concepto, permanecendo como droga de escolha para todas as apresentações clínicas da doença, não havendo relatos consistentes na literatura de resistência treponêmica ao fármaco, além dos benefícios de boa relação custo/efetividade e a não dependência de tecnologia sofisticada (KAMB et al., 2010; REZENDE; BARBOSA, 2015; BRASIL, 2018).

\section{CONSIDERAÇÕES FINAIS}

Considerando o objetivo proposto, observou-se a baixa ocorrência de reações adversas e anafiláticas em gestantes com VDRL positivo após o uso de penicilina benzatina, sendo esta a droga de escolha no tratamento da doença, tendo comprovada a sua eficácia e segurança do uso, ainda que na rede básica de saúde.

Constatou-se que, nesses locais, há maior resistência dos profissionais na disposição do tratamento correto a essas mulheres, especialmente da equipe de enfermagem, por motivos que vão desde a falta de conhecimento acerca da patologia, até a insegurança, permeada pela possibilidade de reações adversas ou anafiláticas, embasada na infraestrutura inadequada das unidades para atender esses casos.

Desse modo, a pesquisa em tela reforça as diretrizes recomendadas, beneficiando a comunidade acadêmico-científica e, principalmente, a população em geral. Além disso, percebeu-se a necessidade de que as organizações de saúde, em parceria com outros setores, promovam e incentivem a realização de capacitações das equipes multiprofissionais sobre o manejo da doença e a importância do seu tratamento adequado e em tempo hábil.

Em conjunto, uma vez que uma das justificativas para a não realização do tratamento na rede básica de saúde seja a falta de estrutura adequada, reiterase a importância do aparato tecnológico, como medicamentos e equipamentos necessários em casos emergenciais, profissionais capacitados, a articulação das redes de saúde que envolvem o sistema de referência e contra referência e o acolhimento adequado dessas demandas.

Para os enfermeiros, que possuem importante papel no controle da sífilis e como membros e líderes de equipes, ao dominarem os conhecimentos necessários, são capazes de orientar, instituir o início precoce do tratamento dessas gestantes, acolhê-las junto de seus parceiros e/ou familiares, estabelecendo uma relação de vínculo, capacitando suas equipes e, com isso, contribuem para a quebra da cadeia de transmissão, melhorando a qualidade de vida do binômio materno infantil.

\section{REFERÊNCIAS}

AVELLEIRA, J. C. R.; BOTTINO, G. Syphilis: diagnosis, treatment and control. Anais Brasileiros de Dermatologia, v. 81, n. 2, p. 111-126, 2006. 
BRACCIO, S.; SHARLAND, M.; LADHANI, S. N. Prevention and treatment of mother-to-child transmission of syphilis. Current Opinion in Infectious Diseases, v. 29, n. 3, p. 268-274, 2016.

BRASIL. Ministério da saúde. Protocolo clínico e diretrizes terapêuticas para prevenção da transmissão vertical de HIV, sífilis e hepatites virais/ Ministério da Saúde, Secretaria de Vigilância em Saúde, Departamento de Atenção à Saúde. - Brasília: Ministério da Saúde, 2018.

CAIADO, J. Hipersensibilidade a fármacos -Tratar, documentar e dessensibilizar. Revista Portuguesa de Imunoalergologia, v. 24, n. 2, p. 111-114, 2016.

COHEN, S. E. et al. Syphilis in the modern era: an update for physicians. Infectious Disease Clinics of North America, v. 27, n. 4, p. 705-722, 2013.

CONSELHO FEDERAL DE ENFERMAGEM. NOTA TECNICA Nº3: Penicilina Benzatina nas Unidades Básicas de Saúde. Brasília, 2017.

DALLÉ, J. et al. Oral Desensitization to Penicillin for the Treatment of Pregnant Women with Syphilis: A Successful Program. Revista Brasileira de Ginecologia e Obstetrícia, v. 40, n. 01, p. 43-46, 2018.

DANTAS, J. D. C. Condutas de profissionais que realizam a consulta prénatal na Estratégia Saúde da Família quanto à detecção, tratamento e acompanhamento da gestante com sífilis. 2008. 112f. Dissertação (Mestrado em Assistência à Saúde) - Universidade Federal do Rio Grande do Norte, Natal, 2008.

DE AQUINO, G. T.; GONÇALVES, H. C. Perfil das mulheres portadoras de sífilis gestacional em Santa Catarina no ano de 2012. Arquivos Catarinenses de Medicina, v. 44, n. 4, p. 72-81, 2016.

DE SANTIS, M. et al. Syphilis infection during pregnancy: fetal risks and clinical management. Infectious Diseases in Obstetrics and Gynecology, 430585, 2012.

FELIX, M. M. R.; MALAMAN, M. F.; ENSINA, L. F. C. Parecer técnico da ASBAI sobre o uso da penicilina $\mathrm{G}$ em unidades básicas de saúde. Brazilian Journal of Allergy and Immunology, v. 2, n. 4, p. 129-131, 2014.

FELIX, M. M. R.; KUSCHNIR, F. C. Alergia à penicilina: aspectos atuais. Revista Adolescência e Saúde, v. 8, n. 3, p. 43-53, 2011.

GALVÃO, T. F. Segurança da benzilpenicilina benzatina na atenção básica em saúde. Boletim Farmacoterapêutica, v. 18, n. 1, 2014.

GROSSI, R. et al. Reação de Jarisch-Herxheimer em gestante com sífilis: relato de caso. Jornal Brasileiro de Doenças Sexualmente Transmissíveis, v. 23, n. 3, p. 149-151, 2011. 
GUANABARA, M. A. O. et al. Acesso de gestantes às tecnologias para prevenção e controle da sífilis congênita em Fortaleza-Ceará, Brasil. Revista de Salud Pública, v. 19, p. 73-78, 2017.

HENZ, C. Sífilis na gestação e desfechos adversos. 2018. 85f. Dissertação (Mestrado em Ciências da Saúde: Ginecologia e Obstetrícia) - Universidade Federal do Rio Grande do Sul, Porto Alegre, 2018.

KALININ, Y.; PASSARELLI NETO, A.; PASSARELLI, D. H. C. Sífilis: aspectos clínicos, transmissão, manifestações orais, diagnóstico e tratamento. Odontologia, v. 23, n. 45-46, p. 65-76, 2016.

KAMB, M. L. et al. A Road Map for the Global Elimination of Congenital Syphilis. Obstetrics and Gynecology International, v. 2, n. 1, p. 1-6, 2010.

NUNES, P. S. et al. Syphilis in pregnancy and congenital syphilis and their relationship with Family Health Strategy coverage, Goiás, Brazil, 2007-2014: an ecological study. Epidemiologia e Serviços de Saúde, v. 27, n. 4, p. 1-10, 2018.

QUÉTEL, C.; BRADDOCK, J.; PIKE, B. History of syphilis. Polity Press Cambridge, 1990.

RAMALHO, M. O. de A. Avaliação da assistência pré-natal com ênfase na sífilis gestacional na estratégia de saúde da família do Recife. 2016.93 f. Dissertação (Mestrado em Saúde da Criança e do Adolescente) - Universidade Federal de Pernambuco, Recife, 2016.

REZENDE, E. M. A.; BARBOSA, N. B. A sífilis congênita como indicador da assistência de pré-natal no Estado de Goiás. Revista de APS, v. 18, n. 2, p. 220 $-232,2015$.

SHENOY, E. S. et al. Evaluation and management of penicillin allergy: a review. Journal of the American Medical Association, v. 321, n. 2, p. 188-199, 2019.

TORRES, R. G. et al. Syphilis in Pregnancy: The Reality in a Public Hospital. Revista Brasileira de Ginecologia e Obstetrícia, v. 41, n. 2, p. 90-96, 2019.

TSAI, S. et al. Syphilis in Pregnancy. Obstetrical \& Gynecological Survey, v. 74, n. 9, p. 557-564, 2019.

TSIMIS, M. E.; SHEFFIELD, J. S. Update on syphilis and pregnancy. Birth Defects Research, v. 109, n. 5, p. 347-352, 2017.

VAZ, M. J. R. Sífilis Congênita: critérios de notificação. Saúde Coletiva, v. 5, n. 25, p. 199-205, 2008. 